\title{
The Effect of Low-Level Light Therapy on Capsaicin- Induced Peripheral and Central Sensitization in Healthy Volunteers: A Double-Blinded, Randomized, Sham-Controlled Trial
}

\author{
Kordula Lang-Illievich • Raimund Winter • Gudrun Rumpold-Seitlinger • \\ Kurt Schicho $\cdot$ Christian Dorn · Christoph Klivinyi · Helmar Bornemann-Cimenti iD
}

Received: August 11, 2020 / Accepted: September 30, 2020 / Published online: October 10, 2020

(C) The Author(s) 2020

\begin{abstract}
Introduction: Several clinical trials have demonstrated that low-level light therapy (LLLT), a method of photobiomodulation, is an effective analgetic treatment. However, the mechanism of action has not yet been finally clarified. In particular, unanswered questions include whether it only affects peripheral or whether it also affects the spinal or supraspinal level. This study aimed to evaluate the effect of low-level light therapy on primary and secondary hyperalgesia in a human pain model.

Methods: This study was planned as a randomized, sham-controlled, and double-blinded trial with repeated measures within subject
\end{abstract}

Significance We present the first data from a human pain model indicating that low-level light therapy effectively modulates both peripheral and central sensitization, making it a promising tool in the management of acute as well as chronic pain.

K. Lang-Illievich · G. Rumpold-Seitlinger ·

C. Dorn - C. Klivinyi · H. Bornemann-Cimenti $(\square)$

Department of Anesthesiology and Intensive Care

Medicine, Medical University of Graz, Graz, Austria

e-mail: helmar.bornemann@medunigraz.at

\section{R. Winter}

Division of Plastic, Aesthetic and Reconstructive

Surgery, Department of Surgery, Medical University

of Graz, Graz, Austria

K. Schicho

Department of Oral and Maxillofacial Surgery,

Medical University of Vienna, Vienna, Austria design. Capsaicin was applied on both forearms of ten healthy volunteers to induce peripheral and central sensitization. One forearm was treated with low-level light therapy; the other served as sham control.

Results: Low-level light therapy significantly increased the mechanical pain threshold, heat pain threshold, and decreased pain intensity.

Conclusions: Our data indicate that low-level light therapy is effective at reducing the heat and mechanical pain threshold in a human pain model, pointing to a significant modulating effect on peripheral and central sensitization. These effects-especially in the absence of reported side effects-make low-level light therapy a promising tool in pain management. The application of low-level light therapy to treat chronic pain should be considered for further clinical trials.

Keywords: Hyperalgesia; Low-level light therapy; Pain sensitization; Photobiomodulation 


\section{Key Summary Points}

Peripheral and central sensitization are pivotal mechanisms in pain physiology.

Peripheral sensitization is often linked to inflammation and acute pain.

Central sensitization is regarded as a key domain in the chronification of pain.

We have shown that low-level light therapy (LLLT) reduces both central and peripheral sensitization.

The effect size of the central effect was even larger than the effect on pain intensity.

Our findings encourage further clinical trials with LLLT in chronic pain.

\section{DIGITAL FEATURES}

This article is published with digital features, including a summary slide, to facilitate understanding of the article. To view digital features for this article go to https://doi.org/10.6084/ m9.figshare.12999494.

\section{INTRODUCTION}

Low-level light therapy (LLLT), a method of photobiomodulation, is well established in various fields of medicine. In general, LLLT is the clinical application of light with wavelengths usually in the range of 600 to $1100 \mathrm{~nm}$ and a typical power density from $5 \mathrm{mWcm}^{-2}$ to $5 \mathrm{Wcm}^{-2}$ [1]. Indications for LLLT are the improvement of wound healing and tissue regeneration, scarring, and perfusion, as well as pain therapy.

In the last few decades, the phenomenon of sensitization has been increasingly studied for its fundamental role in pain medicine $[2,3]$.
Sensitization is generally regarded as a physiological, adaptive, and self-limiting process caused by nociceptive stimuli to facilitate healing of an injury. However, prolonged sensitization is maladaptive and represents a major contribution to the development of pain chronification. Systematically, sensitization can be grouped into central and peripheral mechanisms.

Peripheral sensitization causes the so-called primary hyperalgesia at the nociceptor level due to an excessive stimulus response by decreasing the threshold for nociception in the peripheral nervous system [4]. The heat pain threshold (HPT) is generally regarded as a marker of primary hyperalgesia [5]. In contrast, central sensitization causes secondary hyperalgesia, which is quantified by the mechanical pain threshold (MPT). Central sensitization is determined by the neuroplastic changes occurring at the spinal or supraspinal level [6].

The distinction between these two mechanisms is relevant because peripheral and central sensitization responds to different therapeutic approaches [7]. Clinically, the two are likely to coexist in the majority of patients experiencing pain; in pain chronification, however, the central processes reach major relevance [3]. They play a pivotal role in the development and maintenance of various chronic pain conditions [8]. For example, central sensitization contributes to the transition process from acute to chronic pain and the development of persistent postoperative pain [9].

Despite convincing results in some clinical trials [10-14], the mechanism of action by which LLLT relieves pain has not yet been clarified. In particular, unanswered questions include whether LLLT only takes effect peripherally or whether it also effects the neuroplastic changes on the spinal or supraspinal level.

To provide an answer to these questions, we use a human pain model developed to mimic different aspects of clinical pain and to study pain mechanisms in order to examine whether peripheral or central mechanisms contribute to the analgetic effect of LLLT. 


\section{METHODS}

\section{Study Design and Ethics Statement}

This study was designed as a randomized, shamcontrolled, and double-blinded trial with repeated measures within subject design at the Medical University of Graz, Austria. After receiving approval from the ethics committee (EK 30-270 ex 17/18), healthy male and female subjects aged 20-60 willing to participate were included. Exclusion criteria were defined as: known allergies or hypersensitivity to capsaicin, history of skin diseases, neurological diseases, diabetes mellitus, pregnancy, and consummation of analgetic or psychotropic drugs taken within 1 month before study participation.

Those eligible were informed about the study details by the principal investigator and, after giving written consent, were included in the study. During the first visit, demographic information, including Fitzpatrick classification of skin color, was obtained from all patients [15].

\section{A-Priori Sample-Size Calculation}

No previously published data were available as a source for an a-priori sample-size calculation. Thus, we powered the study to meet the following parameters: the significance level of 0.05 and beta was 0.2 . In a two-sided paired $t$ test, a sample of ten participants is suitable for detecting an effect size of $\geq 1$.

\section{Setting}

The trial took place in a quiet room with an average temperature of $\sim 24^{\circ} \mathrm{C}$ over the whole duration of the capsaicin application and irradiation procedure. Probands were situated in the room for at least $30 \mathrm{~min}$ to allow acclimatization.

\section{Capsaicin Pain Model}

We used a human pain model capable of inducing the central and peripheral mechanisms. Capsaicin application rapidly produces local neurogenic inflammation (characterized by edema and erythema) when locally administered to human skin by stimulating the TRPV1 receptors on dermal sensory nerve endings $[5,16-20]$. In this pain model, heat hyperalgesia (i.e., the increased sensitivity to heat stimulation at the site of application of capsaicin) has been found to be due to peripheral sensitization, whereas mechanical hyperalgesia (i.e., increased sensitivity to mechanical stimulation beyond the area of capsaicin) is due to central sensitization [21].

Before the capsaicin application, skin temperature was measured using an infrared thermometer. In the case of skin temperature below $30^{\circ} \mathrm{C}$, the area was covered by a blanket until the required skin temperature was reached. A $30 \mathrm{~mm} \times 30 \mathrm{~mm}$ cutaneous capsaicin patch $8 \%$ (Qutenza, Grünenthal, Aachen, Germany) with $640 \mathrm{mg}$ of capsaicin per $\mathrm{cm}^{2}$ (corresponding to $5.76 \mathrm{mg}$ of capsaicin in total) was applied in the volar side in the middle of both distal forearms of the participants. The patches were loosely fixed with bandages. After $60 \mathrm{~min}$, the patches were removed, and the exposed skin areas were treated with the appropriate cleansing gel (supplied with Qutenza and intended for removal) according to the manufacturer's recommendations.

\section{Randomization and Blinding}

During the capsaicin application, patients were randomized to determine which arm was treated with LLLT. The other arm received the same treatment with an unactivated device and served as sham-control. Randomization was carried out by a coin toss (left or right) by a nurse, who had no further involvement in the study except for the irradiation procedure. The observer was not present during the randomization and intervention. The nurse was instructed not to share any information about the allocation of the side of irradiation with the volunteers and observers.

The volunteers were blinded with opaque goggles. In addition, they wore earplugs to 
prevent them from hearing the ventilator noise of the LLLT system.

\section{Intervention}

Immediately after removing the capsaicin patch, the two forearms were positioned in the same way on a base divided by an opaque screen to avoid scattered radiation (Fig. 2). The nurse, who was also responsible for the randomization, positioned two identical LLLT devices (Repuls7, Repuls Lichtmedizintechnik GmbH, Vienna, Austria) simultaneously with a $7 \mathrm{~cm}$ distance ring in direct contact with the skin. According to the randomization procedure, one LLLT device remained turned off. The other forearm was irradiated with pulsating and cold red light of $640 \mathrm{~nm}$. The intensity was 175 $\mathrm{mWcm}^{-2}$, which corresponds to a power density of $4100 \mathrm{~mW}$. The pulse frequency was set to $2.5 \mathrm{~Hz}$. A duration of $12 \mathrm{~min}$ was chosen based on the manufacture's recommendations.

\section{Measurement}

Measurement was performed directly after intervention. Pain intensity was quantified using a numeric rating scale (NRS 0-10) immediately after intervention on the irradiated and non-treated forearms. Assessment of the sensory thresholds was based on the specifications of the German Research Association for Neuropathic Pain $[22,23]$.

HPT was assessed on both the LLLT irradiated forearm and non-treated forearm (= control). It was evaluated using a computer-controlled Peltier element, the TSA-II Quantitative NeuroSensory Analyzer (Medoc Ltd., Ramat Yishai, Israel), with a $30 \mathrm{~mm} \times 30 \mathrm{~mm}$ thermode. As heat hyperalgesia is only present in the area of primary hyperalgesia [24], the probe of the TSA was exactly positioned to match the marked edges of the capsaicin patch. The baseline temperature of the probe was $32^{\circ} \mathrm{C}$. The ramp for the temperature increase of the probe was set to $1{ }^{\circ} \mathrm{C} / \mathrm{s}$. The volunteers were instructed to press a button as soon as they felt a sensation that was not only warm but also accompanied by a burning, stinging, drilling, or dragging feeling.
MPT was assessed on both sides $1 \mathrm{~cm}$ proximal to the marked area using pinpricks with a calibrated force of $8,16,32,64,128,256$, and $512 \mathrm{mN}$ and a flat contact area $0.2 \mathrm{~mm}$ in diameter (MRC Systems GmbH, Heidelberg, Germany). The pinpricks were applied with a stepwise which increased force. The volunteers were instructed to indicate the force where they first perceived a sharp sensation. They were then asked about any side effects at the end of the irradiation and the end of the measurement.

\section{Statistical Analysis}

The data were analyzed for normality by the Shapiro-Wilk test. Accordingly, a comparison of the sides was calculated using a paired $t$ test or Wilcoxon signed-rank test, as appropriate. The significance level $\alpha$ was set to 0.05 . To quantify the magnitude of the effect size, Cohen's $d$ for repeated measurement was calculated with their corresponding 95\% confidence interval (95\% $\mathrm{CI})$. The effect sizes were summarized in three categories: small $(0.2<d<0.5)$, medium $(0.5<d<0.8)$, and large $(d>0.8)$ [25].

The force measurements of the mechanical pain sensitivity were transformed into decadic logarithms. Before the logarithmic transformation, a constant of 0.1 was added to all zero and non-zero raw data in order to avoid a loss of zero values [26]. The data were summarized as mean and standard deviations.

All analyses were conducted with NCSS [NCSS 12 Statistical Software (2018); NCSS, LLC. Kaysville, UT, USA]. Data analyses were performed by an independent researcher blinded to the group assignment.

Table 1 Demographic data

\begin{tabular}{ll}
\hline Sex $($ female/male) & $7 / 3$ \\
Age (years) & $41.2 \pm 9.9$ \\
Weight $(\mathrm{kg})$ & $70.1 \pm .8 .3$ \\
Height $(\mathrm{cm})$ & $174.7 \pm 8.5$ \\
\hline
\end{tabular}


This manuscript was prepared according to the Consort guidelines. All procedures were in accordance with the Helsinki Declaration.

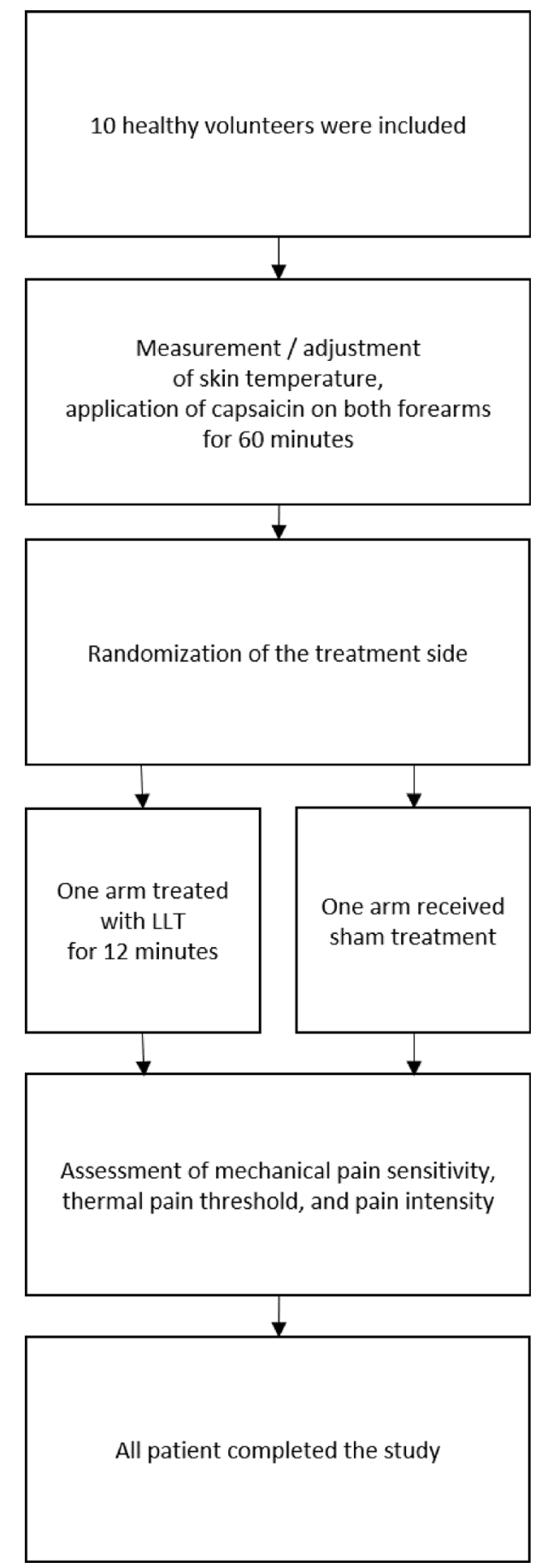

Fig. 1 Study flowchart

\section{RESULTS}

In July and August 2019, seven females and three males were assessed for eligibility and included in the trial. Patient demographics are presented in Table 1. The age of the probands ranged from 27 to 58 years. Eight subjects were right-handed and two were left-handed. All participants were Caucasian and had dark white skin, according to skin type 3 of the Fitzpatrick classification. The treatment side was randomly allocated to the right side in five subjects and to the left side in five subjects. Skin temperature was in the range of $32 \pm 1{ }^{\circ} \mathrm{C}$ before the capsaicin application for all probands without the need for external warming.

A schematic diagram of the time-course of the study is presented in Fig. 1.

NRS pain values differed significantly between the LLLT-treated side and control side (3.6 \pm 1.2 vs. $4.4 \pm 1.1, p=0.0107)$. Likewise, HPT $\quad\left(40.2 \pm 2.5^{\circ} \mathrm{C} \quad\right.$ vs. $36.9 \pm 1.8^{\circ} \mathrm{C}$,

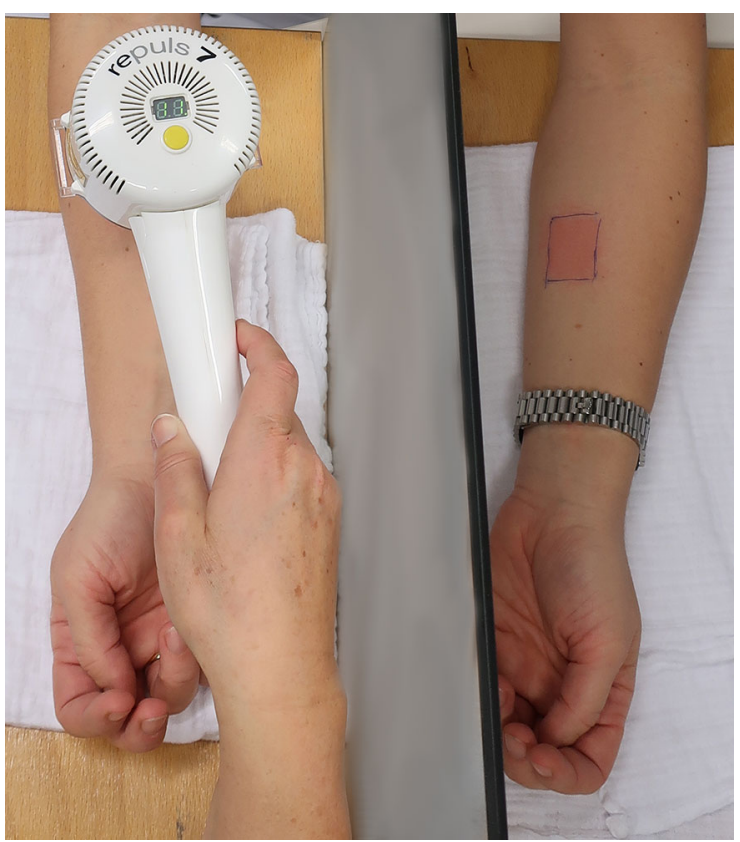

Fig. 2 Setup of the intervention. Both forearms were positioned in the same way on a base divided by an opaque screen to avoid scattered radiation. One side was irradiated and the other side received a sham intervention (not displayed). The red area marks the neurogenic inflammation caused by the capsaicin application 
$p=0.0026)$ and the mechanical pain sensitivity (MPS) $[2.19 \pm 0.43 \log 10(\mathrm{mN})$ vs. $1.89 \pm 0.45$ $\log 10(\mathrm{mN}), p=0.0084]$ differed significantly.

The effect size was 0.682 (95\% CI 0.385-1.03) for the NRS pain values, 1.037 (95\% CI $0.103-1.97)$ for MPS and $1.54 \quad(95 \%$ CI 0.544-2.541) for HPT.

All the participants completed the study. There were no unexpected side effects reported (Fig. 2).

\section{DISCUSSION}

Our study shows that LLLT reduces pain intensity, as well as both peripheral and central sensitization in a human pain model.

\section{Effect on Pain Intensity}

A few clinical trials have demonstrated that LLLT alleviates pain. Most of these studies were carried out in postoperative scenarios. LLLT was shown to be effective in decreasing mean pain intensity in patients undergoing hip arthroplasty [11], coronary bypass surgery [13], and orthodontic tooth movement [10]. Likewise, in a non-surgical acute pain setting, pre-treatment with LLLT before radiotherapy in breast cancer patients had a dual beneficial effect in reducing the severity of radiodermatitis and ameliorating pain intensity due to radiation-induced skin toxicity.

There are very few studies that concentrate on LLLT and chronic pain. In patients with nonspecific chronic knee pain, treatment with LLLT decreased pain and improved quality of life [12].

The results of our trial are in line with the findings of the above-mentioned small number of clinical studies. The pain intensity our probands experienced on the irradiated forearm was reduced by nearly one-quarter compared with the intensity on the sham-treated forearm, representing a medium effect size.

Proposed mechanisms of analgetic action include local release of serotonin, endorphins, and anti-inflammatory effects [13]. Additionally, activation of peripheral opioid receptors may contribute [27].

\section{Effect on Peripheral Sensitization}

Our data have shown that HPT experienced a statistically significant increase in the LLLTtreated forearm compared with the sham-treated forearm. This corresponds to a reduction in peripheral sensitization.

The intracellular mechanisms of sensitization of nociceptors-the structure where the peripheral sensitization mainly takes place-are not yet fully understood. The complex processes of immunology, however, which have recently been well studied, should attract particular attention. Several studies have shown that pronociceptive cytokines like tumor necrosis factor $\alpha$ (TNF- $\alpha$ ), IL-1 $\beta$, and IL- 6 , as well as antinociceptive cytokines like IL-10 contribute to balanced nociceptor sensitivity. LLLT contributes to a decrease in the pro-inflammatory cytokines IL-1 $\beta$ and IL- 6 as well as an increase in the anti-inflammatory cytokine IL$10[28,29]$. In clinical trials, TNF- $\alpha$ and IL-6 showed decreased serum levels after hip arthroplasties were treated with LLLT $[11,30]$. The effect of LLLT on pro-inflammatory TNF- $\alpha$ was studied in a sciatic nerve crush model in mice, but the results were controversial $[29,31]$.

The release of opioid peptides by immune cells is another peripheral endogenous antinociceptive mechanism involved in counteracting inflammatory hyperalgesia [32]. In this context, the LLLT-related activation of peripheral opioid receptors, which involve the recruitment of opioid-containing leukocytes, are certainly of great relevance [27].

\section{Central Sensitization}

Mechanical pain sensitivity differed significantly between the irradiated and non-irradiated forearms, pointing to an influence of LLLT on central sensitization. Although these results may appear counterintuitive at first glance, there are some possible explanations for how changes at the spinal and supraspinal level can be caused by topical treatment. However, as our study was not designed to elucidate the underlying mechanisms, we can only speculate on the potential explanations for our observed 
antihyperalgetic effect. Nitric oxide (NO) is regarded as an important element in central sensitization of nociceptive posterior horn neurons [33-35]. There are references that show the involvement of NO in cellular mechanisms of low-level light therapy $[36,37]$.

Glutamergic calcium conducting ion channels, in particular, NMDA receptor channels, as well as the activation of TRPV1 receptors, are essential for central sensitization processes (i.e., long-term potentiation) [6]. Pigatto et al. demonstrated in a very recent study the involvement of both TRPV1 and glutamate on the analgetic effect of LLLT [38]. One could therefore speculate that these mechanisms may also represent a potential rational for our findings.

\section{Clinical Perspective}

Peripheral and central sensitization are two pivotal mechanisms in our current understanding of pain physiology. While peripheral sensitization is often linked to inflammation, which is a typical component of various acute pain scenarios, central sensitization is regarded as a key domain in the chronification of pain $[2,3]$.

Previous clinical studies on the effectiveness of LLLT have been conducted primarily for the treatment of acute pain $[10,11,13,14]$. These convincing results can be explained by the effect on peripheral sensitization, which we showed in the present study. Our results provide the mechanistic background of the effect of LLLT on acute pain disorders.

What surprised us was the central effect of LLLT. The effect size of these results was even larger than the effect on pain intensity. Until now, the application of LLLT in chronic pain scenarios has only been evaluated in a single clinical trial, which suggested it as an effective treatment option [12]. Our findings encourage further clinical trials on chronic pain.

\section{Limitations}

The present study has limitations that need to be addressed. First, the duration of the LLLT application was chosen arbitrarily. As no previously published evidence was available, we followed the manufacturer's recommendations.

Another limitation is that we applied only a single LLLT treatment. Repeating the LLLT therapy several times would not have been reasonable due to the temporal dynamics of the chosen pain model [39]. However, even our single treatment resulted in medium to strong effects. Evaluation of repeated applications in a prolonged human pain model would thus be theoretically interesting but would raise major ethical issues. Therefore, the effectiveness of different treatment durations should be further studied in clinical scenarios related to chronic pain.

Another interesting parameter would have been the size of the flare area, which occurs as a result of neurogenic inflammation. Unfortunately, we did not have access to the necessary measurement systems for a valid assessment, such as a laser Doppler flowmeter.

Finally, we have to emphasize that this study was not designed for elucidating background mechanisms but to assign the analgetic effect to clinically relevant modes of action. All assumptions on potential underlying causes and contributors are speculative.

\section{CONCLUSIONS}

Our data indicate that LLLT is effective in reducing HPT and MPT in a human pain model, pointing to a significant modulating effect on peripheral and central sensitization. These effects-especially in the absence of reported side effects-make LLLT a promising tool in pain management. The application of LLLT to treat chronic pain should be considered for further clinical trials.

\section{ACKNOWLEDGEMENTS}

We thank the participants of the study and the team of the Interdisciplinary Pain Clinic Graz. 
Funding. This study has been carried out in the course of a research project supported by the Austrian Research Promotion Agency FFG, project no. 853128. Dr. Kurt Schicho has proposed and managed the parts of this project belonging to the field of biomedical engineering.

Authorship. All named authors meet the International Committee of Medical Journal Editors (ICMJE) criteria for authorship for this article, take responsibility for the integrity of the work as a whole, and have given their approval for this version to be published.

Authorship Contributions. KLI: Design of the study, data acquisition, preparing and approving the manuscript. RW: Design of the study, analyzing the data, drafting and approving the manuscript. GRS: Design of the study, data interpretation, drafting and approving the manuscript. KS: Design of the study, data acquisition, drafting and approving the manuscript. CD: Design of the study, data acquisition, drafting and approving the manuscript. CK: Design of the study, data acquisition, drafting and approving the manuscript. HBC: Design of the study, data interpretation, revised and approving the manuscript.

Disclosures. Kordula Lang-Illievich, Raimund Winter, Gudrun Rumpold-Seitlinger, Kurt Schicho, Christian Dorn, Christoph Klivinyi, and Helmar Bornemann-Cimenti have nothing to disclose.

Compliance with Ethics Guidelines. The study protocol was approved by the ethics committee of the Medical University of Graz, Austria (EK 30-270 ex 17/18). The study was carried out in accordance with the Helsinki Declaration. All participants provided informed consent.

Data Availability. The datasets analyzed during the current study are available from the corresponding author on reasonable request.

Open Access. This article is licensed under a Creative Commons Attribution-NonCommercial 4.0 International License, which permits any non-commercial use, sharing, adaptation, distribution and reproduction in any medium or format, as long as you give appropriate credit to the original author(s) and the source, provide a link to the Creative Commons licence, and indicate if changes were made. The images or other third party material in this article are included in the article's Creative Commons licence, unless indicated otherwise in a credit line to the material. If material is not included in the article's Creative Commons licence and your intended use is not permitted by statutory regulation or exceeds the permitted use, you will need to obtain permission directly from the copyright holder. To view a copy of this licence, visit http://creativecommons.org/licenses/by$\mathrm{nc} / 4.0 /$.

\section{REFERENCES}

1. De Freitas LF, Hamblin MR. Proposed mechanisms of photobiomodulation or low-level light therapy. IEEE J Sel Top Quantum Electron. 2016;22:348-64.

2. Gold MS, Gebhart GF. Nociceptor sensitization in pain pathogenesis. Nat Med. 2010;16:1248-57.

3. Woolf CJ. Central sensitization: implications for the diagnosis and treatment of pain. Pain. 2011;152:S2-15.

4. Maihofner C, Nickel FT, Seifert F. Neuropathic pain and neuroplasticity in functional imaging studies. Schmerz. 2010;24:137-45.

5. Vollert J, Magerl W, Baron R, et al. Pathophysiological mechanisms of neuropathic pain: comparison of sensory phenotypes in patients and human surrogate pain models. Pain. 2018;159:1090-102.

6. Jimenez-Andrade JM, Bloom AP, Stake JI, et al. Pathological sprouting of adult nociceptors in chronic prostate cancer-induced bone pain. J Neurosci Off J Soc Neurosci. 2010;30:14649-566.

7. Treede RD, Meyer RA, Raja SN, et al. Peripheral and central mechanisms of cutaneous hyperalgesia. Prog Neurobiol. 1992;38:397-421.

8. Hansen MS, Wetterslev J, Pipper CB, et al. The area of secondary hyperalgesia following heat stimulation in healthy male volunteers: inter- and intraindividual variance and reproducibility. PLOS ONE. 2016;11:e0155284. 
9. Arendt-Nielsen L, Morlion B, Perrot S, et al. Assessment and manifestation of central sensitisation across different chronic pain conditions. Eur J Pain. 2018;22:216-41.

10. Esper MA, Nicolau RA, Arisawa EA. The effect of two phototherapy protocols on pain control in orthodontic procedure-a preliminary clinical study. Lasers Med Sci. 2011;26:657-63.

11. Langella LG, Casalechi HL, Tomazoni SS, et al. Photobiomodulation therapy (PBMT) on acute pain and inflammation in patients who underwent total hip arthroplasty-a randomized, triple-blind, placebo-controlled clinical trial. Lasers Med Sci. 2018;33:1933-40.

12. Leal-Junior EC, Johnson DS, Saltmarche A, et al. Adjunctive use of combination of super-pulsed laser and light-emitting diodes phototherapy on nonspecific knee pain: double-blinded randomized placebo-controlled trial. Lasers Med Sci. 2014;29: 1839-47.

13. Lima ACG, Fernandes GA, Gonzaga IC, et al. Lowlevel laser and light-emitting diode therapy for pain control in hyperglycemic and normoglycemic patients who underwent coronary bypass surgery with internal mammary artery grafts: a randomized, double-blind study with follow-up. Photomed Laser Surg. 2016;34:244-51.

14. Strouthos I, Chatzikonstantinou G, Tselis N, et al. Photobiomodulation therapy for the management of radiation-induced dermatitis. Strahlenther Onkol. 2017;193:491-8.

15. Fitzpatrick TB. Ultraviolet-induced pigmentary changes: benefits and hazards. Curr Probl Dermatol. 1986;15:25-38.

16. Baumann TK, Simone DA, Shain CN, et al. Neurogenic hyperalgesia: the search for the primary cutaneous afferent fibers that contribute to capsaicin-induced pain and hyperalgesia. J Neurophysiol. 1991;66:212-27.

17. Koltzenburg M, Lundberg LE, Torebjörk HE. Dynamic and static components of mechanical hyperalgesia in human hairy skin. Pain. 1992;51: 207-19.

18. Lin $\mathrm{Q}, \mathrm{Wu} \mathrm{J}$, Willis WD. Dorsal root reflexes and cutaneous neurogenic inflammation after intradermal injection of capsaicin in rats. J Neurophysiol. 1999;82:2602-11.

19. Lotsch J, Walter C, Zunftmeister M, et al. A data science approach to the selection of most informative readouts of the human intradermal capsaicin pain model to assess pregabalin effects. Basic Clin Pharmacol Toxicol. 2020;126:318-31.
20. Muley MM, Krustev E, Mcdougall JJ. Preclinical assessment of inflammatory pain. CNS Neurosci Ther. 2016;22:88-101.

21. Zheng Z, Bai L, O'loughlan M, et al. Does electroacupuncture have different effects on peripheral and central sensitization in humans: a randomized controlled study. Front Integr Neurosci. 2019;13: 61.

22. Pfau D, Klein T, Blunk JA, et al. QST-Quantitative Sensorische Testung-Handlungsanweisung für den Untersucher. Lehrstuhl für Neurophysiologie. Mannheim: Universitätsmedizin Mannheim; 2010.

23. Rolke R, Baron R, Maier C, et al. Quantitative sensory testing in the German Research Network on Neuropathic Pain (DFNS): standardized protocol and reference values. Pain. 2006;123:231-43.

24. Ali Z, Meyer R, Campbell JN. Secondary hyperalgesia to mechanical but not heat stimuli following a capsaicin injection in hairy skin. Pain. 1996;68: 401-11.

25. Fritz CO, Morris PE, Richler JJ. Effect size estimates: current use, calculations, and interpretation. J Exp Psychol Gen. 2012;141:2-18.

26. Bartlett MS. The use of transformations. Biometrics. 1947;3:39-52.

27. Cidral-Filho FJ, Mazzardo-Martins L, Martins DF, et al. Light-emitting diode therapy induces analgesia in a mouse model of postoperative pain through activation of peripheral opioid receptors and the Larginine/nitric oxide pathway. Lasers Med Sci. 2014;29:695-702.

28. Laraia EMS, Silva IS, Pereira DM, et al. Effect of lowlevel laser therapy $(660 \mathrm{~nm})$ on acute inflammation induced by tenotomy of Achilles tendon in rats. Photochem Photobiol. 2012;88:1546-50.

29. Martins DF, Turnes BL, Cidral-Filho FJ, et al. Lightemitting diode therapy reduces persistent inflammatory pain: role of interleukin 10 and antioxidant enzymes. Neuroscience. 2016;324:485-95.

30. Li WH, Fassih A, Binner C, et al. Low-level red LED light inhibits hyperkeratinization and inflammation induced by unsaturated fatty acid in an in vitro model mimicking acne. Lasers Surg Med. 2018;50: 158-65.

31. Cidral-Filho F, Martins D, Moré A, et al. Lightemitting diode therapy induces analgesia and decreases spinal cord and sciatic nerve tumour necrosis factor- $\alpha$ levels after sciatic nerve crush in mice. Eur J Pain. 2013;17:1193-204. 
32. Brack A, Labuz D, Schiltz A, et al. Tissue monocytes/macrophages in inflammation: hyperalgesia versus opioid-mediated peripheral antinociception. Anesthesiol J Am Soc Anesthesiol. 2004;101: 204-11.

33. Budzinski M, Misterek K, Gumulka W, et al. Inhibition of inducible nitric oxide synthase in persistent pain. Life Sci. 2000;66:301-5.

34. Hoheisel U, Unger T, Mense S. The possible role of the NO-cGMP pathway in nociception: different spinal and supraspinal action of enzyme blockers on rat dorsal horn neurones. Pain. 2005;117: 358-67.

35. Schmidtko A, Gao W, Konig P, et al. cGMP produced by NO-sensitive guanylyl cyclase essentially contributes to inflammatory and neuropathic pain by using targets different from cGMP-dependent protein kinase I. J Neurosci Off J Soc Neurosci. 2008;28:8568-76.

36. Karu TI, Pyatibrat LV, Afanasyeva NI. Cellular effects of low-power laser therapy can be mediated by nitric oxide. Lasers Surg Med. 2005;36:307-14.

37. Lee HI, Lee SW, Kim SY, et al. Pretreatment with light-emitting diode therapy reduces ischemic brain injury in mice through endothelial nitric oxide synthase-dependent mechanisms. Biochem Biophys Res Commun. 2017;486:945-50.

38. Pigatto GR, Silva CS, Parizotto NA. Photobiomodulation therapy reduces acute pain and inflammation in mice. J Photochem Photobiol B. 2019;196: 111513.

39. Zheng Z, Gibson SJ, Khalil Z, et al. Age-related differences in the time course of capsaicin-induced hyperalgesia. Pain. 2000;85:51-8.

40. Opree A, Kress M. Involvement of the proinflammatory cytokines tumor necrosis factor-alpha, IL-1 beta, and IL- 6 but not IL- 8 in the development of heat hyperalgesia: effects on heat-evoked calcitonin gene-related peptide release from rat skin. J Neurosci Off J Soc Neurosci. 2000;20:6289-93.

41. Wagner R, Janjigian M, Myers RR. Anti-inflammatory interleukin-10 therapy in CCI neuropathy decreases thermal hyperalgesia, macrophage recruitment, and endoneurial TNF-alpha expression. Pain. 1998;74:35-42. 